\title{
Interaction Control and Haptic Feedback for a Lower-limb Rehabilitation Robot with Virtual Environments
}

\author{
Nicholas Berezny ${ }^{1}$, Dariush Dowlatshahi ${ }^{2}$, Mojtaba Ahmadi ${ }^{1}$ \\ ${ }^{1}$ Carleton University \\ 1125 Colonel By Drive, Ottawa, Canada \\ Nicholas.berezny@carleton.ca; mojtabaahmadi@cunet.carleton.ca \\ ${ }^{2}$ University of Ottawa \& Ottawa Hospital Research Institute \\ Ottawa, Canada \\ ddowlat@toh.ca
}

\section{Extended Abstract}

A novel lower-limb rehabilitation robot is presented which targets acute, bed-bound patients. The device incorporates virtual visualizations and games along with haptic feedback to increase user engagement. The creation of this device was primarily motivated by a need to package a previous device, the Virtual Gait Rehabilitation Robot (ViGRR) [1], into a more light-weight and hospital-compatible form factor. Fieldwork was conducted at a local hospital with occupational- and physiotherapists who had experience working with stroke patients. Results indicate that patient engagement is critical, which has also been emphasized in the literature (e.g. [2]). It was also found that most bed-bound exercises focus on simple movements such as knee extension, leg lifts or hip abduction. As such the device only has a single linear DOF actuated using a belt drive, which will be used for proof-of-concept for future devices with more capabilities.

The use of admittance control and haptic coupling is discussed with reference to stability, passivity, and the rendering of force feedback associated with the virtual games. Such control schemes allow for the simulation of a variety of activities or exercises inspired by traditional bed-bound stroke therapy, including sit-to-stand, foot placement and simplified gait training. The use of admittance control, a type of interaction control [3], can provide a programmable amount of assistance in helping the patient move through the exercises. In this way the therapist can tune parameters to the individual so that they receive enough robotic assistance while still requiring their participation and engagement (e.g. without becoming a passive motion machine). Through the haptic coupling introduced by Adams \& Hannaford [4], the controller also allows for the design of complex haptic games to be separated from the design of controller stability, thus allowing for more complex physics engines that can be used to create more complicated and stimulating experiences. Virtual environments can also be used to increase the relevancy of the exercises by simulating real-world activities. The implementation of the controller using open-source technology (Real-time Linux with multithreading) as well as the creation of a wirelessly connected user interface and game visualizations using web technology is also discussed.

Preliminary experiments investigating the functionality of the controller and haptics, as well as the subjective experience of the user, indicate that the device is ready for further testing on acute stroke patients and tests within the hospital environment. Future plans include measuring user engagement and testing within the hospital environment.

\section{References}

[1] K. J. Chisholm, K. Klumper, A. Mullins, M. Ahmadi, “A task oriented haptic gait rehabilitation robot, Mechatronics, vol. 24, no. 8, pp. 1083-1091, 2014.

[2] H. Krebs, B. Volpe, N. Hogan, "A working model of stroke recovery from rehabilitation robotics practitioners," Journal of NeuroEngineering and Rehabilitation, 2009.

[3] N. Hogan, "Impedance Control: An Approach to Manipulation," American Control Conference 1984 (March), pp. 304-313, 1985.

[4] R. J. Adams, B. Hannaford, "Control law design for haptic interfaces to virtual reality," IEEE Transactions on Control Systems Technology, vol. 10, no. 1, pp. 3-13, 2002. 